(Aus dem pharmakologischen Universitätsinstitut in Göttingen.)

\title{
Untersuchungen über die tödliche Adrenalinwirkung am Meerschweinchen.
}

\author{
Von \\ Leo Schmidt.
}

(Eingegangen am 28. Juli 1919.)

\section{Einleitung.}

Zwischen den Experimentalerfahrungen über die pharmakologische Wirkung des Adrenalins und seiner Anwendung in der ärztlichen Praxis ist auch heute noch keine hinreichend breite Brücke geschlagen. Das geht am deutlichsten aus der bedauerlichen Erscheinung hervor, daß die wirksame Substanz der Nebennieren zwar von den Laboratoriumserfahrungen aus gesehen einen ganz außerordentlichen Wirkungsbereich mit Angriffspunkten an den verschiedensten Funktionen des tierischen Organismus besitzt, daß sie aber in der Praxis, zumal außerhalb der Klinik, nur in einem recht bescheidenen Umfang zur Anwendung. kommt.

Die näheren Gründe für diese Frscheinung haben experimentelle Unterlagen insbesondere durch die Untersuchungen von $K \operatorname{retsch} m e^{17}$ ) und Ritz $\operatorname{man} \mathbf{n}^{19}$ ) und die darauf aufbauenden Betrachtungen von Straub ${ }^{20}$ ) erfahren. Durch sie ist in den feineren Wirkungsmechanismus des Adrenalins eine gewisse Klarheit gebracht worden. Stra $\mathbf{u b}^{20}$ ) rechnet das Adrenalin in die Reihe jener Gifte, ,die durch ihre Konzentration im strömenden Blute ihre Wirkung äußern"; er stützt sich dabei auf die Feststellung von Kretsch $\operatorname{mer}^{17}$ ), daß das hervorstechendste Symptom der durch Adrenalin gesetzten Sympathicuserregung, die Blutdrucksteigerung, nur dann und nur so lange zustande kommt, als seine Sekretion ins Blut, d. i. unter experimentellen Bedingungen sein Einströmen von außen her in die Blutbahn, erfolgt, und zwar proportional der Sekretions- (Einströmungs-) Geschwindigkeit. Die ganz analoge Feststellung, die Ritz $\operatorname{man} n^{19}$ ) für eine zweite wichtige Wirkungsäußerung des Adrenalins, die Glykosurie, gemacht hat, dient ihm als weitere Unterlage. Für das Zustandekommen der Adrenalinwirkungen liegen also ganz besondersartige Wirkungsbedingungen vor, und diesen feineren Wirkungsmechanismus des Adrenalins näher zu kennen, würde erst eine Sicherstellung rationeller Ausnutzung seiner Wirkungsmöglichkeiten in der Praxis bedeuten. Daß man von einer solchen Kenntnis noch entfernt ist, geht gerade aus den näheren Erklärungsversuchen hervor, die an die erwähnten drei Veröffentlichungen geknüpft worden sind. Konzentrationsgifte von der Art, wie sich das Adrenalin nach den genannten experimentellen Beobachtungen darstellt, sind nicht selten. Es 
brauoht nur daran erinnert zu werden, daß gerade für die große Gruppe der Narkotica der Fettreihe dieselbe Abhängigkeit der Wirkung von der Konzentration im strömenden Blute besteht. Die besondere Eigentümlichkeit des Konzentrationsgiftes Adrenalin, daB seine Wirkung sehr schnell aufhört, sobald die Konzentration im Blute nicht mehr durch Zutun von außen her (d. h. durch Sekretion bzw. durch Einströmen) aufrechterhalten wird, ist gleichfalls bei den Konzentrationsgiften der Fettreihe verwirklicht, und zwar bei denjenigen unter ihnen, die sehr schnell aus der Blutbahn eliminierbar sind. Die Inhalationsnarkotica, die durch Exhalation schnellstens wieder entfernt werden können, ähneln daher dem Adrenalin mehr als die Hypnotica, die auch nach dem Aufhören des Einströmens noch längere Zeit in merklicher Konzentration kreisen können, weil sie weder durch Exhalation noch durch Exkretion noch auch durch Zerstörung oder durch Bindung so schnell eliminiert werden können. Die erste Besonderheit des Adrenalins liegt also in seiner schnellen Eliminierbarkeit. Worauf diese beruht, ist die erste Unbekannte, mit der die Erklärung seines Wirkungsmechanismus zu rechnen hat. Verfrüht erscheint es, so wie dies seit $S$ tra $\mathrm{u} \mathrm{b}^{29}$ ) geschieht, die Erklärung in einer schnellen Zerstörbarkeit des Adrenalins zu suchen. Man knüpft dąbei an die leichte Oxydationsfähigkeit des Adrenalins in vitro an. Im Organismus indessen spricht nichts für eine schnelle Zerstörbarkeit. Schützt doch schon außerhalb des Organismus Serum* zusatz die Adrenalinlösungen vor der Zerstörung, und haben doch insbesondere Falta und I v covi ${ }^{8}{ }^{8}$ ) dargetan, daß selbst nach Adrenalingaben, die ohne Wirkung bleiben, und selbst bei subcutanem, ja sogar bei peroralem Zuführungsweg unverändertes Adrenalin durch die Niere ausgeschieden wird. Daher entbehrt auch die besondere Annahme, daß Adrenalin gerade in denjenigen Gebilden, an denen es seine Wirkung entfaltet, schnell oder gar vorzugsweise zerstört wird, bisher jeglicher Grundlage. Nur auf Grund dieser Annahme aber könnte diejenige Erklärung, die man seit Straubs Veröffentlichung, offenbar infolge mißverständlicher Auslegung der Straubschen ${ }^{20}$ ) Ausführungen, überall findet, erlaubt erscheinen, die Erklärung nämlich, daß Adrenalin Potentialgiftnatur besitze, daß seine Wirkung also unabhängig sei von der Konzentration im Blute, unabhängig auch von der nach Erreichung des Gleichgewichtes mit dieser Konzentration im Blute erzielten Konzentration in der Zelle, sondern nur zusammenhänge mit dem Vorgang des Einströmens in die Wirkungsstätte, also angewiesen sei auf die Zeiten, in denen noch Ungleichgewicht zwischen Blut- und Zellkonzentration herrsche. Stra ub ${ }^{20}$ ) selbst hat auf diesen wichtigen Unterschied des Adrenalins gegenüber den Potentialgiften aufmerksam gemacht und hervorgehoben, daß bei jedem Potentialgift in den Fällen gleichmäßiger Sekretions- (Einströmungs-) Geschwindigkeit schließlich ein Gleichgewicht zwischen der konstant erhaltenen Blutkonzentration und der Konzentration in der Zelle zustande kommen muß, mit dessen Fintritt die an das Konzentrationsgefälle gebundene Wirkung aufhören muß. Nimmt man etwa an, daß in der Tat das Adrenalin im Organismus im Gegensatz zu anderen Potentialgiften schnell zerstört wird, so ist immer noch zu fragen, an welchen Stellen des Organismus die Zerstörung stattfindet. Spielt sich die Zerstörung im strömenden Blute oder in solchen Zellen ab, die nicht gleichzeitig den Angriffspunkt der Adrenalinwirkung darstellen, so handelt es sich um einen Vorgang, der mit der Gefällewirkung an den Wirkungsstätten nichts zu tun hat. Der Konzentrationsverlust, den die Zerstörung bedingt, wird dann einfach in einem Bereich, der ganz außerhalb der für die Wirkung maßgeblichen Potentialsprungsorte liegt, durch die Sekretion wieder wettgemacht. Glückt dies nicht, überwiegt also die Zerstörungsgeschwindigkeit in diesen ,äußeren“ Zerstörungsstätten, so kann ganz einfach das erforderliche äußere Konzentrationspotential niemals zustande kommen; eine Wirkung ist dann also zu keinem Zeitpunkte der Sekretion möglich. Auch wenn in gleicher Weise ,äußere" und "innere" Zerstörungs- 
prozesse stattfinden, wenn also in gleicher Weise wie das Blut oder unbeteiligte Organe auch die an der Wirkung beteiligten Zellen Adrenalin zerstören, ändert sich noch nichts an diesen Verhältnissen. Die Zerstörungsvorgänge verringern dann gleichlaufend die äußere und die innere Adrenalinkonzentration, gerade das Gefälle zwischen beiden wird also nicht verändert, am allerwenigsten erhöht. Das ändert sich erst, wenn sich die Zerstörung ausschließlich oder wenigstens überwiegend in den Wirkungsstätten abspielt. Dann, aber auch nur dann, ist der Zerstörungsvorgang ein Umstand, der das Zustandekommen eines Konzentrationsgleichgewichtes zwischen außen und innen bei gleichbleibender Sekretion ins Blut verhindert und ein ständiges Konzentrationsgefälle zwischen der konstanten Blutkonzentration und der stets nach Null hinstrebenden Konzentration in derWirkungsstätte aufrechterhält. Diese ganz ausgesuchte Eigenart müßte erst für das Adrenalin dargetan sein, wofern man die Konzentrationsgiftnatur des Adrenalins, $\mathbf{d}$. $\mathbf{h}$. seine von Kretschmer ${ }^{17}$ ) und Ritzmann ${ }^{19}$ ) erwiesene Dauerwirksamkeit bei gleichmäßiger Sekretionsgeschwindigkeit, mit einer behaupteten Potentialgift. natur in Einklang bringen wollte. B a y er ${ }^{1}$ ) macht, wiewohl gerade er die Potentialgiftnatur des Adrenalins besonders hervorhebt und die gänzlich unerwiesene Behauptung aufstellt, daß das Adrenalin ,nur während und nur durch das Ein. dringen in die empfindlichen Zellen wirkt", unbewußt auf diese Ungeklärtheit aufmerksam, indem er zu der Hilfshypothese greifen muß: „Die Zerstörung erfolgt wohl hauptsächlich in den Geweben, und zwar innerhalb der adrenalinempfindlichen Zelle."

Für uns sind also die genannten verdienstvollen Arbeiten, wenn sie auch in der Literatur als die Grundsteine klaren Einblicks in den Wirkungsmechanismus des Adrenalins verwendet werden, vor allem Hinweise auf die Schwierigkeiten einer erfolgreichen Adrenalinapplikation. Schon Kretschmer ${ }^{17}$ ) erschien eine nachhaltige Adrenalinwirkung am besten gesichert durch intravenöse Dauereinflößungen verdünnter Adrenalinlösungen. Wäre das Adrenalin in der Tat ein Potentialgift, so würde es nicht wundernehmen, wenn es auf jedem anderen Zuführungswege schlechter wirksam wäre. Es bedürfte dann nicht einer besonders hohen Zerstörbarkeit, um den Wirksamkeitsverlust bei subcutaner oder peroraler Applikation zu erklären. Der längere Weg vom Unterhautzellgewebe bzw. vom Darm zur Wirkungsstätte könnte den Gefälleverlust und damit die Wirkungsminderung ausreichend erklären. Erst bei einem Konzentrationsgift muß im Falle der Wirkungsminderung durch die Veränderung des Zuführungsweges nach weiteren besonderen Erklärungen gesucht werden. Nun ist aber die Wirkungsminderung bei extravasculärer Zuführung noch keineswegs hinreichend sichergestellt. Die Literaturangaben darüber sind recht widersprechend.

Am deutlichsten geht die Abhängigkeit vom Zuführungsweg aus vergleichenden Untersuchungen hervor, die erst kürzlich von Loewe und Simon ${ }^{14}{ }^{15}$ ) zwischen peroraler und parenteraler Adrenalinzufuhr angestellt wurden. Auch früher schon hatten Falta und Ivcovič ${ }^{8}$ ) gezeigt, daß man per os die ungeheuer hohe Dosis von $150 \mathrm{mg}$ ohne tödliche Wirkung zuführen kann. Die Versuche von Loewe und Simon ${ }^{14},{ }^{15}$ ) ergaben in besonders eindringlicher Weise, daß an Tieren, bei denen die intravenöse Darreichung von $0,4-0,5 \mathrm{mg}$ bereits die höchste Wirksamkeit entfaltet, ja geradezu als eben untertödliche Grenzdosis 
betrachtet werden kann, selbst $40 \mathrm{mg}$ Adrenalin, also die 80 fache Menge, per os noch als nahezu wirkungslos zu betrachten sind. Danach würde sich also das Verhältnis zwischen intravenöser und peroraler Dosis auf 1 zu weit über 80 berechnen.

Um so auffälliger muß es erscheinen, daß für den subcutanen Zuführungsweg die Verhältnisse keineswegs so einfach zu liegen scheinen. Die doch gleichfalls recht breite Kluft, die auf der Grundlage der verbreiteten Potentialgiftannahme zwischen intravenöser und subcutaner Darreichung zu erwarten ist, wird in der experimentellen und in der klinischen Praxis häufig recht gering geachtet.

Allerdings bezeichnet der Praktiker am Menschen sehr verschieden hohe Dosen als erlaubt. Recht zahlreiche Beobachter haben in glaubhaften Erfahrungen am Krankenbett bereits von Dosen unangenehme Nebenwirkungen festgestellt, die nach den Ergebnissen der Tierversuche als sehr gering zu bezeichnen sind. Aber sie machen bei ihrer Empfehlung niedriger Gaben auffälligerweise keinen Unterschied zwischen intravenöser und subcutaner Dosierung. Von $\mathrm{Braun}^{3}$ ) z. B., der bei seiner ausgedehnten Benutzung des Adrenalins in der Lokalanästhesie wohl über eine ganz besonders reiche Erfahrung mit subeutaner Adrenalinzufuhr verfügt, warnt bereits vor einer Überschreitung der subcutanen Gabe von 0,5 mg, einer Dosis also, die bei der intravenösen Zufuhr kaum größeren Bedenken begegnet. Ihm steht ziemlich schroff gegenüber die Angabe von $\mathbf{E}$ okert $t^{5}$ ), der bei diphtheriekranken Kindern oft tagelang bis zu $3 \times 3 \mathrm{mg}$ täglich subcutan verabreicht hat. Er bevorzugt diese Darreichungsweise sogar stark vor der intravenösen, nachdem er mit etwa $0,2 \mathrm{mg}$ intravenös nur flüehtige und unbrauchbare Wirkungen erzielt hat, während die hohen subcutanen Gaben von einwandfreier und bis zu 7 Stunden anhaltender Wirksamkeit waren. Auch Beckers und Meesse $n^{2}$ ) gingen übrigens bereits bis zu $3 \mathrm{mg}$ bei subcutaner Applikation hinauf.

Auch die oben erwähntenUntersuchungen von Loe we und Si mo ${ }^{14,15}$ ) haben für diese Frage nach dem Wirkungsverhältnis zwischen subcutaner und intravenöser Zufuhr zu auffälligen Nebenbefunden geführt. Wenn auch ihre Versuchsreihen zur Beantwortung dieser Nebenfrage nicht groß genug erscheinen, so ergibt sich doch aus ihnen die auffällige Tatsache, da $\beta$ von den drei Versuchstieren, welche $0,5 \mathrm{bzw}$. $0,6 \mathrm{bzw}$. 1,0 mg unter die Rückenhaut eingespritzt erhielten, die beiden letzteren binnen 5 Minuten nach der Adrenalinspritze zugrunde gingen, während bei der Applikation in die Ohrvene in zwei größeren Versuchsreihen Gaben bis zu $0,6 \mathrm{mg}$ anstandslos vertragen wurden. Auch in der früheren tierexperimentellen Literatur findet sich der gleiche Widerspruch für das Verhältnis der subcutanen und der intravenösen Wirksamkeit, wie ihn die obenstehenden Beobachtungen am Menschen dartun. Vielfach (z. B. Loewi, v. Fürth) findet sich die Angabe, daß eine Blutdruckwirkung bei subcutaner Zufuhr überhaupt nicht möglich sei. Diese Behauptung stellt $\mathrm{z}$. B. noch. Ba yer ${ }^{1}$ ) für manche Tierarten auf, und sie scheint eine Stütze zu finden in einer von $\mathrm{Ba} \mathrm{yer}^{\mathbf{1}}$ ) und anderen Autoren, so z. B. auch von Eckert ${ }^{5}$ ), mißverstandenen Angabe Stra $\mathrm{ubs}^{23}$ ). 
Stra ub hebt hervor, daß zur Erzeugung des gleichen Grades von Glykosurie, der bei lange anhaltender $D$ a uerinfusion von $0,06 \mathrm{mg}$ pro $1 \mathrm{~kg}$ in die Vene erzeugt wird, bei e in malig er subcutaner Einverleibung $1 \mathrm{mg}$ pro $1 \mathrm{~kg}$ Kaninchen erforderlich ist. Er selbst errechnet daraus, da B 94\% des subcutan applizierten Adrenalins zerstört worden sind. Wenn er glaubt, für die $\mathrm{Zw}$ wecke dieser Berechnung diese beiden auch abgesehen von der Verschiedenheit des Zuführungsweges sehr verschieden zu bewertenden Applikationen nebeneinanderstellen zu dürfen, so zeigt er damit am deutlichsten, daß er selbst nicht wie diejenigen, die ihn zitieren, die Potentialgiftnatur des Adrenalins behaupten will; denn wenn er Adrenalin für ein Potentialgift hielte, so gäbe es ja, wie bereits oben ausgeführt, zur Deutung eines Unterschiedes in der Wirkungsstärke zwischen der gleichen, einmal subcutan und einmal intravenös applizierten Dosis viel näher liegende Erklärungsmöglichkeiten als die Zerstörung im Unterhautzellgewebe, nämlich einfach den Gefälleunterschied. Nimmt man aber umgekehrt die Zerstörung als Grundlage eines Wirkungsunterschiedes zwischen subkutaner und intravenöser Darreichung, so darf man die beiden von $S t r a \mathrm{ub}^{20}$ ) nebeneinandergestellten Versuche keinesfalls in Parallele setzen. Eine verschiedene Zerstörbarkeit ergäbe sich dann erst, wenn zwischen einmaliger, kurzdauernder Einspritzung ins Unterhautzellgewebe bzw. ins Blut bei gleicher Dosis ein wesentlicher Wirkungsunterschied herauskäme. Denn nehmen wir einmal an, es handle sich um ein einfaches Konzentrationsgift, also etwa um ein Hypnoticum der Fettreihe. Es werde weiter angenommen, daß dieses Hypnoticum bei einmaliger Einspritzung einer hohen Dosis ins Unterhautzellgewebe derart zerstört werde, daß nur $6 \%$ davon zur Wirkung kommen können. Wird die gleiche Dosis auf einmal intravenös injiziert, so wird die Wirkung nicht wesentlich höher ausfallen, wenn die Zerstörbarkeit im Blute genau die gleiche ist wie im Unterhautzellgewebe. Wird aber von dieser auch im Blute zu einem hohen Prozentsatz zerstörbaren Substanz eine wesentlich niedrigere Konzentration dauernd aufrechterhalten, so kann sehr wohl der Einfluß der Zerstörung gegenüber dem dauernd neuen Zustrom so weit in den Hintergrund treten, daß die Dauerwirkung, die bei einmaliger Einspritzung, einerlei ob ins Blut oder ins Unterhautzellgewebe, erst bei sehr hoher Gabe von dem der Zerstörung entgehenden kleinen Bruchteil erzielt wird, von der viel kleineren aber dauernd infundierten Menge bewirkt wird.

Im Hinblick auf die große praktische Bedeutung, die einer klaren Beantwortung der Frage nach dem Verhältnis zwischen subcutaner und intravenöser Adrenalinwirkung zukommt, erschien es wünschenswert, nähere Ermittlungen anzustellen.

Die Schwierigkeiten, die sich der Beantwortung der Frage entgegenstellen, sind allerdings nicht gering. Eine quantitative, zahlenmäßig vergleichbare Bemessung der Adrenalinwirkung ist schwierig. Wählt man z. B. als Kriterium der Adrenalinwirkung die erzielte Blutdrucksteigerung, so kommt man, wie unter anderem vorläufige Vorversuche von Loewe und Kohlfärber gezeigt haben, zu unsicheren Ergebnissen, die denjenigen, bei welchen die tödliche Wirkung am Kaninchen als Maßstab benutzt wird, entgegengesetzte Aussagen machen. Mit anderen Worten: bei subcutaner Applikation mäßiger Adrenalingaben scheint es schwierig, eine Beeinflussung des Blutdrucks am Kaninchen zu erzielen. Dementsprechend sind mir auch in der Literatur nirgends Angaben begegnet, welche zur Beantwortung dieser Frage herangezogen 
werden könnten. Für die erste Orientierung schien daher von vornherein ein Vergleich der tödlichen Dosen empfehlenswert. Aber auch hier bieten sich beträchtliche Schwierigkeiten. Und wenn in der Literatur auch über die tödliche Adrenalindosis nur wenige und zum Teil widersprechende Angaben vorliegen, so ist als Ursache dafür nicht so sehr die mangelnde Aufmerksamkeit der Beobachter und ein ungenügendes Interesse für die Ermittlung dieser Zahlenangaben anzuschuldigen als vielmehr besondere Ungunst der zugrunde liegenden Verhältnisse. Diese erhellt am besten, wenn man zunächst einmal nicht nach der tödlichen Dosis, sondern überhaupt nur nach der Todes u rs a che der unter Adrenalineinfluß zugrunde gegangenen Tiere fragt. Die Tierarten, über die hier Erfahrungen in der Literatur auffindbar sind, sind leider nicht sehr zahlreich.

Cybulski ${ }^{\mathbf{4}}$ ), einer der ersten Untersucher der Wirkungen des Nebennierenextraktes, bemerkte Todesfälle nur bei Kaninchen. Auch Oliver und Schäfer ${ }^{18}$ ), denen wir die ersten Forschungen über die Pharmakologie der Nebennierenextrakte verdanken, beobachteten nur an Kaninchen tödliche Wirkung. Gluzinski ${ }^{11}$ ) beschreibt als Wirkungen der Nebennierenzufuhr am Kaninchen Parese, Anästhesie der hinteren Extremitäten, in der vorderen Hälfte leichte Zuckungen, manchmal ausgesprochenen Opisthotonus, Beschleunigung der Atmung und $\mathrm{Pu}$ pillenerweiterung. Nach seiner Beschreibung tritt unter Steigerung der dyspnoischen Erscheinungen und unter allgemeiner Lähmung der Tod ein. Bei der Sektion finden sich starkes Lungenödem und Extravasate, die er hauptsächlich in der Pleura und dem Perikard nachweisen kann. Von anderen Säugetieren gibt GI u zinski1) nur an, daß sie entweder Schwächeerscheinungen aufwiesen, die bald zur Norm zurückkehrten, oder daß sie unter Abmagerung nach einigen Tagen zugrunde gingen.

Auch Szy monowic $z^{21}$ ) erwähnt nur beiläufig tödliche Wirkungen, bei denen die Sektion Extravasate in Lunge, Herz, Gehirn und Medulla oblongata, ja sogar Infarkte und Lungenödem ergab. Was die tödlichen Dosen dieser älteren Untersucher anbelangt, so lassen sie sich schon deswegen nur schwer fassen, weil von ihnen nicht reines Adrenalin, sondern nur wässerige oder glycerin-wässerige Extrakte der Drüse verwendet wurden. Aber schon für diese unexakte Prüfung wird von den Autoren die Ungleichmäßigkeit der tödlichen Dosis hervorgehoben. Gaben, die bei einzelnen Kaninchen tödlich wirkten, konnten bei anderen ohne Lebensgefahr bedeutend überschritten werden.

Als dann das wirksame Prinzip der Nebenniere näher erforscht (von Fürth, Abel, Takamine, Alderich), in seiner Konstitution erkannt und auch synthetischer Reinherstellung zugänglich gemacht worden war, fand die Beschäftigung mit der neuen Reinsubstanz in der Analyse ihrer Angriffspunkte und ihres Wirkungsmechanismus verlockende Aufgaben. Daher finden sich auch in der seitdem gehäuften Adrenalinliteratur stets nur gelegentlich eingestreute, ganz beiläufige Angaben über tödliche Dosen, Todesursachen und Abhängigkeit vom Zuführungsweg. So gibt z. B. Lesage ${ }^{13}$ ) gelegentlich als tödliche Dosis 0,1 bis $0,2 \mathrm{mg}$ für das Kaninchen an, während Exner $\mathrm{r}^{7}$ intraperitoneal 0,5, ja sogar $0,6 \mathrm{mg}$ Adrenalin ohne Lebensgefahr anwenden konnte. Zeig a $\mathrm{n}^{22}$ ) konnte in den Duralsack bis zu $3 \mathrm{mg}$ Adrenalin einspritzen, ohne daß die behandelten Katzen zugrunde gingen. Fröhlich und $\mathrm{Pick}^{9}$ ) konnten einem Hund von $16 \mathrm{~kg}$ mehrfache Gaben von $20 \mathrm{mg}$ unter hochgradiger Blutdruckwirkung einverleiben, wenn 
sie das befürchtete Lungenödem durch Atropin verhüteten. Am ausführlichsten in dieser Literatur, die an den hier aufgeworfenen Fragen so achtlos vorüberging, hat sich $\mathrm{Erb}^{6}$ ) gelegentlich seiner klassischen Nachprüfung der Adrenalinarterionekrose mit dem Studium der Todesdose und Todesursache des Adrenalins beim Kaninchen befaßt. Er kommt nicht zu einer einheitlichen Zahl für die tödliche Dosis und stimmt hierin mit dem überein, worauf auch die Ermittlungen anderer Autoren schon hinweisen. Er hebt hervor, daß einerseits Tiere schon nach $0,05 \mathrm{mg}$ pro $1 \mathrm{~kg}$ Körpergewicht verendeten, andererseits 0,3 mg pro $1 \mathrm{~kg}$ Körpergewicht gut vertragen wurden. Übersieht man die Gesamtheit seiner Versuche, so finden sich darin sogar solche, in denen bis zu 1,1 mg pro $1 \mathrm{~kg}$ Körpergewicht ohne Schädigung vertragen wurde. Auch die Todesursache ist nach seinen Versuchen keine einheitliche. Die Tiere sterben keineswegs regelmäßig an einem Lungenödem, von welchem Fröhlich und $\mathrm{Pick}^{9}$ ) beim Hund die einzige Todesgefahr fürchteten. Auch da, wo der Tod unter Lungenödem eintrat, fand er größere oder kleinere hämorrhagische Infarkte in der Lunge. In anderen Fällen traten die tödlichen Hämorrhagien ohne Infarzierung oder Ödemisierung der Lungen in ganz anderen Körperbereichen, so z. B. in einem seiner Fälle als retroperitoneale Blutung auf. Selbst über die gleichfalls nicht unwichtige Frage, ob mehrfache Vorbehandlung verstärkend oder abschwächend wirkt, geben seine Versuche keine eindeutige Auskunft. In einzelnen Fällen gelang es ihm durch kurz aufeinanderfolgende Einzelinjektionen die Widerstandsfähigkeit in ganz analoger Weise, wie dies später Fröhlich und $\mathrm{Pick}^{9}$ ) beim. Hunde glückte, außerordentlich hochzutreiben. Auch bei Intervallen von 24 und mehr Stunden trat häufig ein Rückgang der Empfindlichkeit auf. Mehrfach betont er, daß seine je einmal täglich behandelten Tiere nach einiger Zeit auf ein Mehrfaches der Anfangsdosis mit schwächeren Vergiftungserscheinungen reagierten, als sie die erste niedrig bemessene Injektion ausgelöst hatte. Auf der anderen Seite weist er aber auch Versuche auf, in denen er genötigt war, von einer höheren Dosis allmählich wieder auf eine niedrigere herunterzugehen. So mußte er z. B. in einem Falle eine bis auf 0,7 hochgetriebene Gabe wieder bis auf $0,1 \mathrm{mg}$ zurückstellen, wobei schließlich das Tier selbst auf $0,1 \mathrm{mg}$ stärker reagierte als im Anfange der Behandlung auf ein Mehrfaches dieser Dosis.

Alles in allem stellen sich also nach diesen Literaturangaben die Aussichten, exakte Auskunft über das Verhältnis zwischen subcutaner und intravenöser Wirksamkeit des Adrenalins zu erhalten, nicht besonders günstig dar. Man gewinnt vielmehr den Eindruck, als ob das Adrenalin auch in hohen Gaben nur funktionelle Änderungen setzt, die an sich niemals lebensbedrohend sind. Tritt der Tod ein, so rührt das nur daher, daß ein Locus minoris resistentiae gegenüber dieser veränderten Funktion vorhanden ist. Die Durchlässigkeit der Lungengefäße liefert keineswegs mit Regelmäßigkeit die Unterlage für eine solche tödliche Sekundärwirkung des Adrenalins beim Kaninchen. Bei dieser Tierart scheint vielmehr eine von Individuum zu Individuum ihren Grad und ihre Lokalisation im Verlauf der Gefäßbahn wechselnde Zerreißlichkeit der Gefäßwand das zum Sekundärtode führende Moment darzustellen. Damit variiert aber auch die Lebensgefahr, die mit einer solchen Ruptur verbunden ist, und die Dosis, die zu einer tödlichen Blutung führt. Wenn ich mir daher zur Aufgabe setzte, die Wirksamkeit subcutaner und intravenöser Adrenalingaben zu vergleichen und an 
dem Maßstabe der tödlichen Dosis für jeden dieser beiden Zuführungswege zu messen, so war nach alledem klar, daß das Kaninchen kein geeignetes Versuchsobjekt darstellen konnte und daß auch von den anderen in der Literatur erwähnten Tierarten keine den dort zu findenden Angaben zufolge günstigere Verhältnisse erwarten ließ.

Leider verboten mir die Zeitumstände, daß ich eine größere Reihe von Tierarten in der angedeuteten Richtung untersuchte. Die herrschenden Schwierigkeiten in der Beschaffung von Tiermaterial geboten von vornherein die äußerste Sparsamkeit und legten daher starke Beschränkung für alle derartige Versuche auf, in denen der Tod des Versuchstieres als Indicator benutzt wird. Glücklicherweise gelang es mir, mir trotz der bestehenden Beschaffungschwierigkeit wenigstens eine gewisse Zahl von Meerschweinchen für derartige Versuche verfügbar zu machen. An ihnen konnte ich zunächst die Frage prüfen, ob auch diese Tierart in der tödlichen Wirkung des Adrenalins ebenso stark wie die Kaninchen von den individuellen Zufälligkeiten eines wechselnden Locus minoris resistentiae abhängig ist. Schon bald stellte sich durch die Ubereinstimmung der Sektionsbefunde heraus, daß bei der von mir gewählten Tierart die Todesursache nach Adrenalineinspritzung eine wesentlich einheitlichere ist, und so konnte ich mir mit größerer Aussicht auf Erfolg, als dies bei der Verwendung von Kaninchen möglich gewesen wäre, die weitere Frage vorlegen, welche zahlenmäßigen Beziehungen zwischen der tödlichen Grenzdosis für den subcutanen und derjenigen für den intravenösen Zuführungsweg bestehen. Auch auf die Frage nach der Bedeutung einer Vorbehandlung mit Adrenalin brauchte bei der Einheitlichkeit der Todesursache nicht eine so vieldeutige Antwort befürchtet $\mathrm{zu}$ werden, wie sie $\operatorname{Erbs}^{6}$ ) Kaninchenversuche gaben. Es schien daher am Platze, auch diese dritte Frage in den Bereich meiner Prüfung einzubeziehen.

\$ Ich faßte diese dritte Fragestellung so, daß ich zu ermitteln versuchte, ob bei dem Adrenalin, dessen Wirkung ja bei kurzzeitiger Einspritzung an eine verhältnismäßig kurze Zeitdauer geknüpft ist, eine zweite nach längerer oder kürzerer Frist erfolgende Einspritzung von genau der gleichen Wirksamkeit wie die erste Injektion ist. Ein solcher Einfluß konnte in zwei Richtungen zutage treten, wenn er an einer Verschiebung der tödlichen Grenzdosis gemessen wurde. Diese konnte erhöht werden, wenn eine Empfindlichkeitsverminderung durch vorausgegangene Adrenalinbehandlung herbeigeführt wird, wie das ja vor allem Fröhlich und $\mathrm{Pick}^{9}$ ) am Hunde, $\mathrm{Erb}^{6}$ ) an seinen beiden in einstündigen Abständen mit Adrenalin gespritzten Kaninchen zu erhärten scheinen.

Umgekehrt mußte auch damit gerechnet werden, daß die tödliche Dosis nach einer Vorbehandlung verringert war. Dann war auch ohne 
nähere Analyse der Schluß erlaubt, daß über die vorübergehende funktionelle, prompt reversible Wirkung hinaus eine länger anhalténde Schädigung durch die erste Injektion gesetzt wird, auf welche sich dann die Wirksamkeit einer zweiten untertödlichen Gabe in kumulierender Weise aufpfropft.

\section{Experimenteller Teil.}

Subcutane Injektion.

Zunächst wurde die tödliche Grenzdosis für das Meerschweinchen bei subcutaner Zuführung ermittelt. Zu den Injektionen wurde durchweg Suprareninlösung (Höchst) 1: 1000 verwandt, die frisch von der Apotheke bezogen wurde. Die Injektion erfolgte unter die Rückenhaut. Die verwendeten Meerschweinchen waren sämtlich gesunde Tiere, die kurz zuvor vom gleichen Züchter bezogen waren.

Die ausführlichen Protokolle dieser Versuche finden sich im Anhang als Versuchsprotokolle Nr. 1-21. Die Ergebnisse finden sich in der nachfolgenden Tabelle $I$ in übersichtlicher Form zusammengestellt. Bis zu einer Dosis von $0,7 \mathrm{mg}$ pro $1 \mathrm{~kg}$ Körpergewicht einschließlich werden die Einspritzungen anstandslos vertragen. Die Vergiftungserscheinungen bei den höheren dieser Dosen entsprechen im großen und ganzen der Beschreibung von Gluzinski11) für das Kaninchen. Nachdem das Tier einige Minuten ohne irgendwelche auffälligen Krankheitserscheinungen verblieben ist und höchstens eine langsame und leicht verflachte Atmung aufgewiesen hat, beginnt es allmählich Atemfrequenz und Tiefe zu steigern. Gleichzeitig beginnt sich eine gewisse Schwäche der Extremitäten geltend zu machen. Die Vorderbeine stützen den Rumpf nicht mehr, sondern gleiten seitlich ab und lassen die Brust auf die Unterlage sinken. Häufig beginnen die Schwächeerscheinungen an den hinteren Extremitäten, die meist bei einem leichten Fortbewegungsversuch nachschleifen und das Tier mit nach hinten gestreckten Hinterbeinen auf den Bauch sinken lassen. Sehr selten kam bei diesen Wirkungsgraden passive Seitenlage zustande. Meist äußerte sich der Gipfel der Wirkung nur in äußerst frequenter und vertiefter Atmung, wobei der ganze Körper erschüttert wird und der Kopf im Rhythmus der Atmung ruckende Bewegungen ausführt. Sieht man von dem bei $0,72 \mathrm{mg}$ pro $1 \mathrm{~kg}$ Körpergewicht zugrunde gegangenen Tier ab, welches schon vorher einen kranken Eindruck machte, so ändern sich die Erscheinungen auch bis zu einer Gabe von $0,8 \mathrm{mg}$ pro $1 \mathrm{~kg}$ Körpergewicht nicht. 0,8-0,84 mg pro $1 \mathrm{~kg}$ Körpergewicht dürfen wohl nach meiner Versuchsreihe als die unterste tödliche Grenzdosis betrachtet werden. Von höheren Gaben darf regelmäßig tödliche Wirkung erwartet werden. Die Rücksichtnahme auf das spärliche Tiermaterial zwang mich, von einer Erhärtung dieser 
naheliegenden Vermutung Abstand zu nehmen. Auch bei den zugrunde gegangenen Tieren verliefen die Vergiftungserscheinungen zunächst in der oben beschriebenen Weise, doch führten in dem Stadium der Extremitätenschwäche und Atemstörungen weitere Fortbewegungsversuche regelmäßig zu passiver Seitenlage, in der das Tier dann unter zunehmender Atemstörung, jedoch bis gegen das Ende hin ohne Bewußtseinsstörung, verharrt. Häufig, jedoch nicht regelmäßig, schlossen sich Krampferscheinungen an, die zuweilen das Tier in heftigster Weise auf der Unterlage herumwarfen. Nach hochgradiger Frequenzvermehrung ging die Atmung gewöhnlich in eine Phase über, die durch zunehmende

Tabelle I. Subcutane Injektion.

\begin{tabular}{|c|c|c|}
\hline Versuch & $\begin{array}{c}\text { Dosis } \\
\text { mg pro } \mathrm{kg}\end{array}$ & Erfolg \\
\hline Nr. 1 & 0,20 & erholt \\
\hline , $\quad 2$ & 0,30 & , \\
\hline , 3 & 0,50 & ", \\
\hline , $\quad 4$ & 0,50 & , \\
\hline$" 5$ & 0,50 & " \\
\hline$"$ & 0,60 & ", \\
\hline ," & 0,65 & , \\
\hline , & 0,65 & ", \\
\hline , & 0,68 & ," \\
\hline$\Rightarrow 10$ & 0,70 & ", \\
\hline , 11 & 0,72 & tot \\
\hline,$\quad 12$ & 0,75 & erholt \\
\hline$\Rightarrow 13$ & 0,78 & , \\
\hline,$\quad 14$ & 0,78 & , \\
\hline , 15 & 0,80 & ", \\
\hline, 16 & 0,80 & " \\
\hline,$\quad 17$ & 0,80 & tot \\
\hline , 18 & 0,82 & erholt \\
\hline , 19 & 0,84 & tot \\
\hline „, 20 & 1,50 & $\because$ \\
\hline
\end{tabular}

Vertiefung aber abnehmende Frequenz gekennzeichnet ist. Die letzten außerordentlich tiefen Atemzüge rückten dann bis zu 12 Minuten langem Intervall auseinander. Stets überlebte das Herz den letzten Atemzug. Häufig entleerte das Tier bei den letzten Atemzügen plötzlich aus der Nase reichliche Mengen fast reinen, nur wenig schaumigen Blutes, das auch nach dem Tode in deutlich tropfendem Strome noch kurze Zeit nachträufelte.

Nicht selten begannen diese Entleerungen aus der Nase schon wesentlich früher, bisweilen bereits vor dem eigentlichen Krampfstadium. Je eher sie einsetzten, desto weniger bluthaltig war die entleerte Flüssigkeit. Häufig begannen diese Erscheinungen damit, daß die Exspiration 
von einigen feinen Spritzern ganz schwach sanguinolent verfärbter Flüssigkeit begleitet war. Allmählich entleerte sich dann etwas mehr schwach rosa gefärbter Schaum und erst dann rieselte immer mehr und immer unverdünnteres Blut aus den Nasenlöchern.

Abweichende Erscheinungen, wie etwa Hinweise auf Blutungen im Bereich des Zentralnervensystems oder andere außerhalb des Bereiches der Lunge gelegene Hämorrhagien, konnte ich beim Meerschweinchen nie beobachten. Dieser Einheitlichkeit der Symptome entspricht auch der Sektionsbefund. Stets überlagerte die Lunge in mehr oder weniger ausgiebiger Weise das Herz, stets war sie in mehr oder weniger gleichmäßiger Weise von großen frischen Hämorrhagien durchsetzt und stets war das Lungengewicht wesentlich vermehrt. Utber das Durchschnittsgewicht der normalen Meerschweinchenlunge liegen Literaturangaben nicht vor. Doch sind im hiesigen Institut bei anderem Anla B Ermittlungen hierüber angestellt worden, deren Kenntnis ich Herrn Professor Loewe verdanke. Danach weicht das Gewicht der Meerschweinchenlunge von demjenigen anderer genauer untersuchten Tierarten nicht wesentlich ab. Während das Normalgewicht der Katzenlunge auf etwa $8 \mathrm{~g}$ pro $1 \mathrm{~kg}$ Körpergewicht angesetzt wird, dasjenige der Kaninchenlunge sich zwischen 3 und $5 \mathrm{~g}$ pro $1 \mathrm{~kg}$ Körpergewicht bewegt, dürfte das der Meerschweinchenlunge ungefähr in der Mitte zwischen beiden Tierarten liegen. Man begeht jedenfalls sicher keine Unterschätzung des Lungengewichts, wenn man die normale Meerschweinchenlunge auf höchstens $8 \mathrm{~g}$ pro $1 \mathrm{~kg}$ Körpergewicht veranschlagt.

Intravenöse Zufuhr.

Um die tödliche Dosis für das Meerschweinchen bei Einspritzung in die Blutbahn zu ermitteln, wurden die Tiere auf einem Operationsbrett aufgespannt und nach Freilegung der Vena jugularis mit einer Tuberkulinspritze mit scharfer Kanüle injiziert. Die Ergebnisse dieser intravenösen Injektionsversuche sind im einzelnen den Versuchsprotokollen Nr. 21 bis 27 (Anhang, Seite $303 \mathrm{ff}$.) zu entnehmen. Sie finden sich außerdem in der folgenden Tabelle II.

Tabelle II. Intravenöse Injektion.

\begin{tabular}{c||c|c}
\hline Versuch & $\begin{array}{c}\text { Dosis } \\
\text { mg pro kg }\end{array}$ & Erîolg \\
\hline \hline Nr. 21 & 0,057 & erholt \\
, 22 & 0,085 & ", \\
, 23 & 0,15 & tot \\
, 24 & 0,20 &, \\
", 26 & 0,20 & ", \\
, 27 & $<0,50$ &,
\end{tabular}


Wie aus der Tabelle ersichtlich, ist die Grenze der tödlichen Wirkung eine recht scharfe. Von $0,15 \mathrm{mg}$ pro $1 \mathrm{~kg}$ Körpergewicht an sterben die Tiere regelmäßig. Darunter tritt kein Todesfall mehr ein. Die tödliche Grenzdosis liegt also zwischen 0,085 und 0,15 mg pro $1 \mathrm{~kg}$ Körpergewicht. Um den Einfluß der Konzentration auszuschalten, der bei dieser kleinen Tierart für die Lösung 1: 1000 immerhin in Rechnung zu ziehen ist, haben wir von $0,2 \mathrm{mg}$ ab nach unten die Konzentration um eine Größenordnung niedriger gewählt.

Die Vergiftungserscheinungen waren bei allen Tieren die gleichen wie bei den subcutan behandelten, ja auch die Zeit zwischen Injektion und Tod wird durch die intravenöse Zufuhr nicht merklich abgekürzt. Der Tod tritt nach intravenöser Zufuhr 6-12 Minuten nach der Einspritzung ein. Der subcutane Zuführungsweg verzögert ihn nicht, denn hier sterben die Tiere, wenn man von den beiden Ausnahmefällen des Spättodes nach mehr als einer Stunde absieht, 6-13 Minuten nach der Injektion. Siehe Tab. IV am Schluß des experimentellen Teils. Auch die Sektionsbefunde sind, wie aus der Tabelle IV hervorgeht, ganz genau die gleichen wie nach subcutaner Zufuhr.

Das Verhältnis zwischen subcutaner und intravenöser Dosis beträgt demnach für das Meerschweinchen, gemessen an der jeweils tödlichen Grenzdosis, etwa $8: 1$.

Wiederholte Subcutanbehandıung.

Nachdem sich so in den beiden vorausgehenden Abschnitten ergeben hatte, daß bei der von mir gewählten Tierart für die subcutane wie auch für die intravenöse Adrenalinzufuhr eine hinreichend scharfe, individuell wenig schwankende tödliche Grenzdosis besteht, konnte mit ëiniger Aussicht auf Erfolg daran gegangen werden, die unsicheren Ergebnisse, welche die Studien über den Einfluß einer Adrenalinbehandlung an anderen Tierarten gezeitigt hatten, einer Nachprüfung zu unterziehen.

Ich wählte folgenden Untersuchungsmodus: Steigenden Dosen von Adrenalin wurden in steigendem Zeitintervall jeweils zweite Gaben von Adrenalin nachgeschickt. In einer Anzahl der Versuche wurde wiederum in passendem Abstand eine dritte, schließlich sogar eine vierte Adrenalindosis angefügt. Für alle Gaben, die Vor- wie die Nachbehandlung, wurde der subcutane Zuführungsweg gewählt. Die Intervalle wurden zwischen $1 \frac{1}{2}$ und 24 Stunden variiert.

Nachdem sich in denjenigen Versuchen, in denen der Erfolg einmaliger Nachbehandlung geprüft wurde, ein unverkennbarer Einfluß der Vorbehandlung herausgestellt hatte, beschränkte ich mich für die Versuche mit mehrfacher Nachbehandlung auf die Prüfung in dem größeren, für die praktische Seite der Frage zunächst in Betracht kommenden Intervall von 24 Stunden. 
Die Versuchsprotokolle dieses Abschnittes finden sich im Anhang, S. $304 \mathrm{ff}$, die übersichtliche Zusammenstellung ihrer Ergebnisse in der nachfolgenden Tabelle III.

Tabelle III. Wiederholte subcutane Injektion.

\begin{tabular}{|c|c|c|c|c|c|c|c|c|}
\hline Versuch & $\begin{array}{l}\text { I. Dosis } \\
\text { mg pro } \mathrm{kg}\end{array}$ & $\begin{array}{l}\text { Inter- } \\
\text { vall } \\
\text { Std. }\end{array}$ & $\begin{array}{l}\text { II. Dosis } \\
\mathrm{mg} \text { pro } \mathrm{kg}\end{array}$ & $\begin{array}{c}\text { Inter- } \\
\text { vall } \\
\text { Std. }\end{array}$ & $\begin{array}{l}\text { III. Dosis } \\
\text { mg pro kg }\end{array}$ & $\begin{array}{l}\text { Inter- } \\
\text { vall } \\
\text { Std. }\end{array}$ & $\begin{array}{l}\text { IV. Dosis } \\
\text { mg pro kg }\end{array}$ & Erfolg \\
\hline Nr. 28 & 0,2 & $11 / 2$ & 0,3 & - & - & - & - & erholt \\
\hline , 29 & 0,2 & $1 \frac{1}{2}$ & 0,4 & - & - & - & - & ", \\
\hline,$\quad 30$ & 0,2 & 2 & 0,3 & 一 & - & - & - & ", \\
\hline , 31 & 0,2 & 2 & 0,3 & $\ldots$ & - & - & - & tot \\
\hline , 32 & 0,2 & 2 & 0,4 & - & - & - & - & erholt \\
\hline , 33 & 0 & 3 & 0,3 & - & - & - & - & ", \\
\hline,$\quad 34$ & 0,2 & $4^{1 / 2}$ & 0,73 & - & - & - & - & tot \\
\hline,$\quad 35$ & 0,3 & 2 & 0,2 & - & - & - & - & erholt \\
\hline „, 36 & 0,3 & 3 & 0,2 & - & $\ldots$ & - & - & , \\
\hline,$\quad 37$ & 0,3 & $\mathbf{4}^{1 / 2}$ & 0,6 & - & - & 一 & - & tot \\
\hline,$\quad 38$ & 0,5 & $4^{1} / 2$ & 0,45 & - & - & - & - & ", \\
\hline , $\quad 39$ & 0,5 & 24 & 0,5 & - & - & - & - & erholt \\
\hline , 40 & 0,5 & 24 & 0,55 & - & - & - & - & ", \\
\hline , 41 & 0,5 & 24 & 0,55 & 24. & 0,55 & - & - & ", \\
\hline " 42 & 0,5 & 24 & 0,5 & 24 & 0,6 & - & - & $\begin{array}{l}\text { tot } n . \\
48 \mathrm{Std} .\end{array}$ \\
\hline , 43 & 0,5 & 24 & 0,55 & 24 & 0,55 & 24 & 0,63 & tot \\
\hline
\end{tabular}

Wie sich aus den Angaben der Tabelle III entnehmen läßt, macht sich ein ungünstiger Einfluß der Vorbehandlung auf die Wirkung der zweiten Adrenalingabe deutlich geltend. Daß nicht etwa eine einfache Kumulierung im exakten Sinne, wie sie die Digitaliskörper aufweisen, vorliegt, zeigt die Unregelmäßigkeit des Versuchsausfalls. Es gelingt zunächst keineswegs, wie etwa bei dem Digitoxin, eine erste halbfach tödliche Dosis durch eine zweite, ebenso hohe zur tödlichen Wirkung zu ergänzen. Auch kurze Zeitintervalle sind dazu nicht ausreichend. Dagegen zeigt sich deutlich in dreien von den vier tödlich endenden Versuchen dieser Serie, daß noch im Abstande von $\mathbf{4}^{\mathbf{1}} / \mathbf{2}$ Stunden eine Ergänzung der weit untertödlichen ersten Gabe $(0,2$ bzw. 0,3 bzw. $0,5 \mathrm{mg})$ durch eine zweite, gleichfalls unterhalb der tödlichen Grenzdosis liegende $(0,73$ bzw. 0,6 bzw. $0,45 \mathrm{mg})$ zum Tode ausreicht. In allen diesen drei Fällen beträgt die so in zwei Sitzungen einverleibte Gesamtdosis nicht viel mehr als die für einmalige Einspritzung ermittelte tödliche Grenzdosis $(0,9$ bzw. 0,93 bzw. $0,95 \mathrm{mg}$ gegenüber $0,8-0,84 \mathrm{mg}$ bei einmaliger Applikation).

Rückt man mit den beiden Gaben noch näher zusammen, so scheint der ungünstige Einfluß der Vorbehandlung noch verstärkt zu werden. Wenigstens spricht hierfür der beachtenswerte Ausfall des Versuches 
Nr. 30, in welchem bei Zwischenschaltung eines Intervalles von nur 2 Stunden bereits eine Gesamtgabe von $0,5 \mathrm{mg}$ pro $1 \mathrm{~kg}$, also nicht viel mehr als die Hälfte der tödlichen Grenzdosis, zum Tode führte. Freilich muß hervorgehoben werden, daß dieser ungünstige Ausgang solch niedriger Gaben nicht regelmäßig erfolgt. In einem zweiten Versuche mit gleicher Anordnung trat der Tod des Versuchstieres nicht ein, wenn es auch auffallend schwere Nacherscheinungen aufwies, und in einem dritten Versuch mit gleichem Zeitabstand, aber sogar etwas erhöhter Gesamtdoșis blieb gleichfalls der tödliche Ausgang aus. Zerlegung einer bedeutend untertödlichen Gesamtdosis in zwei durch Intervalle von $4^{1} / 2$ oder weniger Stunden getrennte Teilgaben kann also diese niedere Gabe zu tödlichem Erfolg führen, doch kommt ein derart ungünstiger Einfluß nicht regelmäßig zustande. Wird das Intervall auf 24 Stunden erhöht, so ist der ungünstige Einfluß der Vorbehandlung noch weiter verringert, er verschwindet indessen, wie die beiden letzten Versuche der Tabelle zeigen, auch dann noch keineswegs vollständig. Eine Gabe, die als einmalige niemals zum Tode führte $(0,6 \mathrm{mg}$, also $3 / 4$ der tödlichen Grenzdosis) wird zur tödlichen, wenn sie am dritten Tag einer in 24 stündigem Abstand vorausgeschickten Behandlung mit zweimal je 0,5 mg nachfolgt. Und ebenso führt die kaum höhere Gabe von 0,63 als vierte Gabe einer ebensolchen Serienbehandlung nach Vorbehandlung mit drei Einzelgaben von 0,5 bzw. $0,55 \mathrm{mg}$ zum Tode.

Der Sektionsbefund ergibt, daß in diesen adrenalinvorbehandelten Fällen nicht etwa eine Wirkung an neuem Angriffspunkt des Adrenalins kumuliert wird. Die klinischen Erscheinungen wie auch der Lungenbefund der nach Vorbehandlung zum Exitus gekommenen Tiere zeigt, daß auch in diesen Fällen wiederholter Behandlung der Angriffspunkt der tödlichen Wirkung derselbe ist, den diese bei einmaliger Behandlung bei unserer Tierart mit Regelmäßigkeit wählt. Auch in diesen unseren Fällen, in denen eine niedrigere Gabe zum Tode führt, wird der Tod durch Lungenerscheinungen, und zwar durch Ödem und multiple Hämorrhagien herbeigeführt. Wenn hierzu nach ein- oder mehrmaliger Vorbehandlung in kürzerem oder auch längerem Intervall wesentlich kleinere Gaben genügen, so ist die Annahme berechtigt, daß die Wirkungsweise der Vorbehandlung in einer vorbereitenden Wirkung am gleichen Angriffspunkte besteht. Auch ohne die histologische Nachprüfung der Lungen dieser Tiere darf vielleicht angenommen werden, daß die vorausgegangene Behandlung mit $1 / 4-5 / 8$ der tödlichen Grenzdosis bereits zu beträchtlichen;, wenn auch längst nicht tödlichen Lungenerscheinungen geführt hat, deren Nachwirkungen auch nach 24 Stunden noch imstande sind, den Boden dafür zu liefern, daßein nachfolgender weiterer, gleichfalls an sich nicht zum 'Tode führender Erguß von Blut und Ödemflüssigkeit in andere Partien der Lunge sich mit ihr zur Erstickungswirkung addiert. 
Adrenalin - Atropinkombination beim Meerschweinchen.

Das Meerschweinchen bietet nach allen meinen bisherigen ${ }^{-}$Versuchen ganz besonders günstige Verhältnisse für das Studium einzelner an die Adrenalinwirkung zu knüpfender Fragen. Der Tod tritt hier im Vergleich zu anderen mit ganz wechselndem Angriffspunkt durch Adrenalin lebensgefährdeten Tieren in sehr einheitlicher Weise durch schwere, multiple Hämorrhagien im Bereich der ganzen Lunge ein. Die Disposition der einzelnen Meerschweinchenindividuen zu derartigen Lungenhämorrhagien von seinem den Erstickungstod bewirkenden Umfang ist gleichfalls eine überraschend einheitliche. Nun geht aus der Literatur hervor, daß in manchen Fällen die tödliche Adrenalinwirkung durch kurz vorausgeschickte Atropingaben verhütet werden kann. Ich knüpfe hier insbesondere an die Angaben von Pick und Fröhlich ${ }^{9}$ ) an. Ihnen gelang es, durch mehrfache vorherige Atropingaben von $5 \mathrm{mg}$ bei einem $16 \mathrm{~kg}$ schweren Hunde die tödliche Wirkung mehrmals wiederholter Injektionen von Adrenalinmengen bis zu $20 \mathrm{mg}$ auszuschalten. Nach ihren Angaben ist beim Hunde die Lebensgefahr solcher hohen Adrenalingaben in dem zur Erstickung führenden Lungen öde m zu suchen. Ich habe daher anhangsweise einige Versuche der Frage gewidmet, inwieweit auch beim Meerschweinchen etwa ein derartiger Antagonismus zwischen Atropin und Adrenalin in Erscheinung tritt.

Die beiden einschlägigen Versuche sind ausführlicher im Anhang auf S. 306 wiedergegeben. Ihr wesentlichstes Ergebnis ist, daß nach im Abstand von jeweils 30 und 10 Minuten vorausgeschickten Atropingaben von je 0,2 bzw. 0,18 und $0,15 \mathrm{~g}$ die übertödliche Dosis von 1,0 bzw. sogar 1,5 mg Adrenalin subcutan nicht nur ohne tödlichen Erfolg, sondern sogar überhaupt mit auffallend abgeschwächter Wirkung verläuft.

Dieses Versuchsergebnis ist um so auffälliger, als nach allen unseren Sektionsbefunden ein Lungenödem beim Zustandekommen der tödlichen Wirkung am Meerschweinchen zum mindesten keineswegs den alleinigen Ausschlag gibt. Wenn vielleicht auch durch histologische Analysen das Lungenödem stärker hervortreten würde, so genügen doch die aus dem makroskopischen Sektionsbefund sich ergebenden ausgedehnten Hämorrhagien vollkommen, um einen Erstickungstod durch die Blutung allein ohne Mitwirkung eines Ödems hinreichend zu erklären.

Die Erklärung für die antagonistische Wirksamkeit des Atropins gegenüber dem Adrenalin beim Meerschweinchen wird durch diese Verhältnisse wesentlich erschwert. Sie muß besonderen Untersuchungen vorbehalten bleiben. Ich beschränke mich darauf, die Tatsache zu verzeichnen, daß auch bei dieser Tierart und auch bei dieser besonderen 
Gestaltung der tödlichen Wirkungsweise des Adrenalins Atropin eine außerordentlich günstige antidotarische Wirkungsweise entfaltet.

Tabelle IV.

Ubersicht der tödlioh verlaufenen Vergiftungsversuche.

\begin{tabular}{|c|c|c|c|c|c|c|c|}
\hline Versuch & Gewicht & $\begin{array}{c}\text { Art der } \\
\text { Behandlung }\end{array}$ & $\begin{array}{c}\text { Zahl der } \\
\text { Einzelgaben }\end{array}$ & $\begin{array}{c}\text { Blutung } \\
\text { aus der Nase }\end{array}$ & $\begin{array}{l}\text { Lungen- } \\
\text { gewicht } \\
\text { pro } \mathrm{kg}\end{array}$ & \multicolumn{2}{|c|}{$\begin{array}{c}\text { Zeitabstand } \\
\text { zwischen Injek- } \\
\text { tion und Tod }\end{array}$} \\
\hline Nr. 11. & $360 \mathrm{~g}$ & subcutan & 1 & nein & 14 & & Min. \\
\hline$\Rightarrow 15$ & 380, & $"$ & 1 & ja & 21 & 10 & ", \\
\hline$\Rightarrow \quad 19$ & 390, & , & 1 & ,, & 25 & 7 & $"$ \\
\hline , 20 & 360, & ", & 1 & , & 20 & 7 & ", \\
\hline , 23 & 350, & intravenös & 1 & ", & 23 & 12 & $"$ \\
\hline ", 24 & $360 "$ & , & 1 & , & 20 & 10 & $"$ \\
\hline,$\quad 25$ & 450, & , & 1 & , & 24 & 8 & , \\
\hline,$\quad 26$ & 520, & $"$ & 1 & ", & 25 & 6 & $"$ \\
\hline , 27 & 540, & ", & 1 & , & 10 & 10 & , \\
\hline , 30 & 520 & subcutan & 2 & , & 11 & 10 & ", \\
\hline ,, 34 & 470 & " & 2 & " & 24 & 6 & $"$ \\
\hline ", 37 & 465, & , & 2 & "? & 20 & 10 & $"$ \\
\hline ", 38 & 390, & , & 2 & nein & 13 & 13 & ", \\
\hline , 42 & 395, & ", & 3 & ", & - & nach 4 & 8 Std. \\
\hline , 43 & 490 & $"$ & 4 & $"$ & 14 & 111 & Min. \\
\hline
\end{tabular}

\section{Zusammenfassung.}

Die tödliche Wirkung subeutaner und intravenöser Adrenalingaben kommt beim Meerschweinchen auf besonders einheitlicher Grundlage zustande. Die Todesursache ist stets in multiplen Lungenhämorrhagien zu finden, welche - vielleicht unter Mitwirkung eines Lungenödems ohne hervortretende individuelle Empfindlichkeitsschwankungen von einer bestimmten, hinreichend scharfen Grenzdosis an den Erstickungstod herbeiführen.

Die tödliche Grenzdosis für das Meerschweinchen liegt nach subcutaner Zufuhr bei etwa $0,8 \mathrm{mg}$ Adrenalin pro $1 \mathrm{~kg}$, nach intravenöser $\mathbf{Z u}$ fuhr zwischen 0,08 und $0,15 \mathrm{mg}$ pro $1 \mathrm{~kg}$. Bei dieser Tierart verhält sich also der Wirkungsgrad intravenöser Zufuhr zu demjenigen der Applikation ins Unterhautzellgewebe wie etwa 1:8.

Adrenalinvorbehandlung steigert im Gegensatz zu den widersprechenden Beobachtungen an anderen Tierarten die Adrenalinempfindlichkeit des Meerschweinchens. Bei Zerlegung in zwei durch ein Intervall von $4^{1} / 2$ oder weniger Stunden getrennte Teilgaben kann schon eine Gesamtdosis zum Tode führen, welche die tödliche Grenzgabe für einmalige Applikation nur wenig überschreitet. Ja, sogar bei einer Gesamtgabe, die wesentlich unterhalb der einmaligen tödlichen Grenzdosis verbleibt, ist bei einem Intervall von 2 Stunden bereits tödliche Wirkung. 
möglich. Auch wenn die Abstände zwischen den Einzelgaben 24 Stunden betragen, ist die Vorbehandlung mit untertödlichen Dosen noch von ungünstiger Nachwirkung, indem auch in solcher Serienbehandlung untertödliche Dosen schließlich zum Tode führen können.

Der Sektionsbefund solcher nach Vorbehandlung zum Tode gekommener Tiere legt die Vermutung nahe, daß untertödliche Dosen bereits zu Lungenblutungen führen, so daß eine neue, durch Adrenalin gesetzte multiple Hämorrhagie dann leichter das Leben gefährdet.

Auch bei dieser Tierart, bei der nicht Lungenödem, sondern hämorrhagischer Lungeninfarkt die tödliche Adrenalinwirkung bedingt, entfaltet Atropin eine sehr augenfällige antagonistische Wirksamkeit.

\section{Anhang.}

\section{Versuchsprotokolle. \\ Subcutane Injektion.}

Versuch 1. Meerschweinchen: Gewicht $470 \mathrm{~g}$.

Injektion von $0,1 \mathrm{ccm}$ Suprareninlösung $1: 1000=0,2 \mathrm{ccm}$ pro $\mathrm{kg}$ K.-G. Beobachtung: Keine von der Norm abweichende Erscheinung.

Versuch 2. Meerschweinchen: Gewicht $465 \mathrm{~g}$.

Injektion von $0,15 \mathrm{ccm}$ Suprareninlösung 1: $1000=0,3 \mathrm{ccm}$ pro $\mathrm{kg} \mathrm{K}$.G. Beobachtung: Wie in Versuch 1.

Versuch 3. Meerschweinchen: Gewicht $390 \mathrm{~g}$.

Injektion von $0,2 \mathrm{~cm}$ Suprareninlösung $1: 1000=0,5 \mathrm{ccm}$ pro $\mathrm{kg} \mathrm{K}$.G. Beobachtung: Nichts Besonderes.

Versuch 4. Meerschweinchen: Gewicht $490 \mathrm{~g}$.

Injektion von $0,25 \mathrm{ccm}$ Suprareninlösung $1: 1000=0,5 \mathrm{ccm}$ pro $\mathrm{kg} \mathrm{K}$.G. Beobachtung: Leichte Erregbarkeit, verstärkte Atmung, Unruhe.

Versuch 5. Meerschweinchen: Gewicht $395 \mathrm{~g}$.

Injektion von $0,2 \mathrm{ccm}$ Suprareninlösung $1: 1000=0,5 \mathrm{~cm}$ pro kg K.-G.

Beobachtung: Leichtes Erregungsstadium. Kehrt innerhalb 20 Minuten zur Norm zurück.

Versuch 6. Meerschweinchen: Gewicht $380 \mathrm{~g}$.

Injektion von $0,23 \mathrm{ccm}$ Suprareninlösung $1: 1000=0,6 \mathrm{ccm}$ pro $\mathrm{kg} \mathrm{K}$.G.

Beobachtung: Wird nach 4 Minuten sehr unruhig, Atmung beschleunigt, vertieft. Ist in seinen Bewegungen nicht mehr sicher. Die binteren Extremitäten gleiten aus, so daß bei Laufversuchen der Körper des Tieres auf der Unterlage hin- und herrutscht. Nach einigen Minuten beginnende langsame Erholung. Die Bewegungen sind aber noch unsicher. Kehrt innerhalb von 40 Minuten zur Norm zurück.

Versuch 7. Meerschweinchen: Gewicht $385 \mathrm{~g}$.

Injektion von $0,25 \mathrm{ccm}$ Suprareninlösung $1: 1000=0,65 \mathrm{~cm}$ pro $\mathrm{kg}$ K. $-\mathrm{G}$.

Beobachtung: Das Tier sitzt zuerst ganz ruhig da, Atmung ist flach und langsam. Allmählich sich steigernde Unruhe. Atmung wird frequenter. Die Bewegungen sind unsicher, das Tier liegt mit dem Bauch auf der Unterlage auf, die hinteren Extremitäten nach hinten abgestreckt. Erholt sich nach 20 Minuten wieder etwas, 
ist in seinen Bewegungen aber noch sehr unsicher. Nach einer $1 / 2$ Stunde noch leicht krank.

Versuch 8. Meerschweinchen: Gewicht $460 \mathrm{~g}$.

Injektion von 0,30 ccm Suprareninlösung l: $1000=0,65$ pro kg K.-G.

Beobachtung: Zeigt die Erscheinungen des vorhergehenden Tieres, jedoch die Schwäche in den hinteren Extremitäten nicht so ausgeprägt.

Versuch 9. Meerschweinchen: Gewicht $430 \mathrm{~g}$.

Injektion von $0,29 \mathrm{ccm}$ Suprareninlösung $1: 1000=0,68 \mathrm{ccm}$ pro $\mathrm{kg} \mathrm{K}$.G.

Beobachtung: Während der ersten 3 Minuten ruhige Atmung, dann tritt Steigerung der Atemfrequenz ein. Das Tier zeigt leichte Erregung, die hinteren Extremitäten sind halb gelähmt. Erscheinungen gehen in der Zeit von einer $1 / 2$ Stunde vorïber.

Versuch 10. Meerschweinchen: Gewicht $350 \mathrm{~g}$.

Injektion von $0,24 \mathrm{ccm}$ Suprareninlösung $1: 1000=0,7 \mathrm{~cm}$ pro $\mathrm{kg} \mathrm{K}$.-G.

Beobachtung: Tier bietet dieselben Erscheinungen wie das vorhergehende.

Versuch 11. Meerschweinchen: Gewicht $360 \mathrm{~g}$.

Injektion von $0,26 \mathrm{ccm}$ Suprareninlösung $1: 1000=0,72 \mathrm{cem}$ pro kg K.-G.

Beobachtung: Sitzt während der ersten Minuten ruhig da, wird dann sehr unruhig, atmet sehr schnell und stoßend. Macht einen schwerkranken Eindruck. Stirbt nach $1 \frac{1}{2}$ Stunden, blutiger Schaum wird nicht entleert. (War schon vorher krank.)

Sektionsbefund: Lunge überlagert leicht das Herz. Lunge ist leicht ödematös, zahlreiche Hämorrhagien. Gewicht der Lunge 5 g

Versuch 12. Meerschweinchen: Gewicht $430 \mathrm{~g}$.

Injektion von $0,32 \mathrm{ccm}$. Suprareninlösung $1: 1000=0,75 \mathrm{ccm}$ pro $\mathrm{kg} \mathrm{K}$.G.

Beobachtung: Macht zunächst einen kranken Eindruck, bietet dieselben Erscheinungen wie die vorhergehenden Versuchstiere. Erholt sich aber bald wieder.

Versuch 13. Meerschweinchen: Gewicht $360 \mathrm{~g}$.

Injektion von $0,28 \mathrm{ccm}$ Suprareninlöşung 1: $1000=0,78 \mathrm{ccm}$ pro $\mathrm{kg}$ K. -G.

Beobachtung: Wie in Versuch 12.

Versuch 14. Meerschweinchen: Gewicht $720 \mathrm{~g}$.

Injektion von $0,56 \mathrm{com}$ Suprareninlösung $1: 1000=0,78 \mathrm{ccm}$ pro $\mathrm{kg} \mathrm{K}$.G.

Beobachtung: Wie in Versuch 12.

Versuch 15. Meerschweinchen: Gewicht $380 \mathrm{~g}$.

Injektion von $0,3 \mathrm{ccm}$ Suprareninlösung $1: 1000=0,8 \mathrm{ccm}$ pro $\mathrm{kg} \mathrm{K.G}$.

Beobachtung: Nach einigen Minuten beginnt das Tier sehr unruhig zu werden, die Atmung ist beschleunigt, Atemzüge vertieft. Die Bewegungen sind unsicher, die Extremitäten gleiten bei Laufversuchen aus. Nach 8 Minuten fällt das Tier auf die Seite, bleibt in passiver Seitenlage liegen, Lauf- und Atemkrämpfe treten auf, blutiger Schaum wird aus der Nase entleert. Nach 10 Minuten tritt unter zunehmenden Atemkrämpfen der Tod ein.

Sektionsbefund: Rechte Lunge überlagert weit das Herz, Lunge stark ödematös, zahlreiche frische Hämorrhagien. Trachea mit blutigem Schaum gefüllt. Gewicht der Lunge 8 g.

Versuch 16. Meerschweinchen: Gewicht $290 \mathrm{~g}$.

Injektion von $0,23 \mathrm{ccm}$ Suprareninlösung $1: 1000=0,8 \mathrm{~cm}$ pro $\mathrm{kg} \mathrm{K}$.-G.

Beobachtung: Das Tier bietet die gleichen Erscheinungen wie die vorhergehenden Versuchstiere.

Versuch 17. Meerschweinchen: Gewicht $350 \mathrm{~g}$.

Injektion von $0,28 \mathrm{ccm}$ Suprareninlösung $\mathrm{I}: 1000=0,8 \mathrm{ccm}$ pro $\mathrm{kg} \mathrm{K}$.-G. 
Beobachtung: Wie in Versuch 16.

Versuch 18. Meerschweinchen: Gewicht $450 \mathrm{~g}$.

Injektion von $0,37 \mathrm{ccm}$ Suprareninlösung $1: 1000=0,82 \mathrm{ccm}$ pro kg K.-G.

Beobachtung: Macht sehr bald einen schwerkranken Eindruck. Die Atmung ist sehr frequent und oberflächlich. Es tritt Schwäche der hinteren Extremität ein. Bei Fortbewegungsversuchen wird der Hinterkörper nachgeschleift. Erholung tritt nach ungefähr 40 Mintuen ein.

Versuch 19. Meerschweinchen: Gewicht $390 \mathrm{~g}$.

Injektion von $0,33 \mathrm{ccm}$ Suprareninlösung $1: 1000=0,84 \mathrm{ccm}$ pro $\mathrm{kg} \mathrm{K}$.-G.

Beobachtung: Beginnt nach wenigen Minuten unruhig zu werden, Atmung stark beschleunigt, stoßweise. Der Kopf folgt ruckweise den rhythmischen Atembewegungen. Rasseln in der Trachea. Fällt auf die Seite, bleibt in passiver Seitenlage liegen, Laufkrämpfe treten auf. Nach 11 Minuten erfolgt unter zunehmender Dyspnöe der Exitus. Danach fließt reines Blut aus der Nase.

Sektionsbef und: Lunge tiefdunkel marmoriert, stark ödematös, zahlreiche Hämorrhagien. Uberlagert weit das Herz. Extravasate finden sich in der Pleura und im Perikard. Gewicht der Lungen $9 \frac{1}{2} \mathrm{~g}$.

Versuch 20. Meerschweinchen: Gewicht $360 \mathrm{~g}$.

Injektion von $0,5 \mathrm{ccm}$ Suprareninlösung $1: 1000=1,5 \mathrm{ccm}$ pro $\mathrm{kg} \mathrm{K}$.-G.

Beobachtung: Tier zeigt dieselben Erscheinungen wie das vorhergehende Versuchstier. Bei der stoßweisen Atmung werden Bluttröpfchen aus der Nase entleert. Rasseln in der Trachea. Nach 7 Minuten tritt der Tod ein. Danach fließt reichlich reines Blut aus der Nase.

Sektionsbefund: Der gleiche wie beim vorhergehenden Versuchstier. Gewicht der Lunge $7 \mathrm{~g}$.

\section{Intravenöse Injektion.}

Versuch 21. Meerschweinchen: Gewicht $365 \mathrm{~g}$.

Injektion von $0,002 \mathrm{ccm}$ Suprareninlösung $1: 1000=0,057 \mathrm{ccm}$ pro $\mathrm{kg} \mathrm{K}$. -G.

Die Dosis wird mit Ringerlösung 10 fach verdünnt.

Beobachtung: Tier ist etwas erregt.

Versuch 22. Meerschweinchen: Gewicht $360 \mathrm{~g}$.

Injektion von $0,03 \mathrm{ccm}$ Suprareninlösung $1: 1000=0,085 \mathrm{ccm}$ pro kg K.-G. Die Dosis wird mit Ringerlösung 10 fach verdünnt.

Beobachtung: Nach 3 Minuten beschleunigte Atmung, Unruhe, Unsicherheit, Taumeln. Nach 7 Minuten Atmung höchst beschleunigt, etwa nach jedem zehnten Atemzuge eine Bauchinspiration. Tier erholt sich langsam.

Versuch 23. Meerschweinchen: Gewicht $350 \mathrm{~g}$.

Injektion von $0,05 \mathrm{ccm}$ Suprareninlösung $1: 1000=0,15 \mathrm{ccm}$ pro kg K.-G. Die Dosis wird mit Ringerlösung 10 fach verdünnt.

Beobachtung: Tier taumelt nach 2 Minuten. Die Extremitäten gleiten aus, Atmung wird flach und langsam. Nach 8 Minuten zuckende Bewegungsversuche, nach 10 Minuten wird blutiger Schaum aus der Nase entleert, nachdem die bisher krampfhaften aber spontanen Zuckungen zugenommen haben. Tier stirbt unter zunehmender Atemnot und Erregung nach 12 Minuten.

Sektionsbefund: Wie bei den vorhergehenden Tieren. Gewicht der Lunge $8 \mathrm{~g}$.

Vers uch 24. Meerschweinchen: Gewicht $360 \mathrm{~g}$.

Injektion von 0,07 ccm Suprareninlösung $1: 1000=0,2 \mathrm{ccm}$ pro $\mathrm{kg} \mathrm{K} . \mathrm{G}$. Die Dosis wird mit Ringerlösung 10 fach verdünnt. 
Beobachtung: Nach 7 Minuten Laufkrämpfe und plötzliche Entleerung von schaumigem Blut aus der Nase. Nach 10 Minuten der letzte Atemzug.

Sektionsbef und: Herz nicht überlagert, Lunge nicht so stark marmoriert wie bei den beiden vorhergehenden Tieren. Trachea entleert blutigen Schaum. Gewicht der Lunge $7 \mathrm{~g}$.

Versuch 25. Meerschweinchen: Gewicht $450 \mathrm{~g}$.

Injektion von $0,09 \mathrm{ccm}$ Suprareninlösung $1: 1000=0,2 \mathrm{ccm}$ pro $\mathrm{kg} \mathrm{K}$.G.

Beobachtung: Zuerst treten Streckkrämpfe auf, dann Laufkrämpfe. Nach 7 Minuten wird tiefdunkelroter Schaum aus der Nase entleert. Vereinzelte Zuckungen der hinteren Extremitäten. Reines Blut fließt aus der Nase. Nach 8 Minuten tritt der Tod ein.

Sektionsbef und: Herz überlagert. Lunge stark vergrößert, ödematös, dunkelrot marmoriert. Gewicht der Lunge $11 \mathrm{~g}$.

Versuch 26. Meerschweinchen: Gewicht $520 \mathrm{~g}$.

Injektion von $0,2 \mathrm{~cm}$ Suprareninlösung $1: 1000=0,4 \mathrm{~cm}$ pro $\mathrm{kg} \mathrm{K}$.-G.

Beobachtung: Atmet zuerst ruhig, dann hört man Rasseln in der Trachea. Tier wird bei zunehmendem Atmen immer unruhiger. Blutiger Schaum wird aus der Nase entleert. Nach 6 Minuten fließt reines Blut aus der Nase. Exitus.

Sektionsbef und: Wie bei Versuchstier 25. Gewicht der Lunge $13 \mathrm{~g}$.

Vers uch 27. Meerschweinchen: Gewicht $540 \mathrm{~g}$. K.-G.

Injektion von $0,27 \mathrm{ccm}$ Suprareninlösung $1: 1000=<0,5 \mathrm{ccm}$ pro $\mathrm{kg}$

Beobachtung: Stirbt binnen 10 Minuten unter zunehmenden Atemkrämpfen. Blutiger Schaum, dann reines Blut wird aus der Nase entleert.

Sektio ns bef u nd: Herz nicht überlagert. Lunge weniger ödematös. Weniger zahlreiche Hämorrhagien. Lungengewicht $5 \mathrm{~g}$.

Wiederholte Subcutaninjektionen.

Versuch 28. Meerschweinchen: Gewicht $350 \mathrm{~g}$.

Injektionen von $0,2 \mathrm{~cm}$, nach $1 \frac{1}{2}$ Stunden von $0,3 \mathrm{ccm}$ pro $\mathrm{kg} \mathrm{K}$.G.

Die Dosis wird mit Ringerlösung 10 fach verdünnt.

Beobachtung: Das Tier macht zunächst einen kranken Eindruck, erholt sich dann aber wieder.

Versuch 29. Meersehweinchen: Gewicht $370 \mathrm{~g}$.

Injektionen von $0,2 \mathrm{ccm}$, nach $1 \frac{1}{2}$ Stunden von $0,4 \mathrm{ccm}$ pro $\mathrm{kg} \mathrm{K}$.G.

Die Dosis wird mit Ringerlösung $10 \mathrm{mal}$ verdünnt.

Beobachtung: Wie in Versuch 28.

Versuch 30. Meerschweinchen: Gewicht $520 \mathrm{~g}$.

Injektionen von $0,2 \mathrm{ccm}$ pro $\mathrm{kg} \mathrm{K}$.-G., nach 2 Stunden von $0,3 \mathrm{ccm}$ pro $\mathrm{kg} \mathrm{K}$.-G.

Beobachtung: Nach 4 Minuten fällt das Tier ohne vorhergehende Erscheinungen plötzlich auf die Seite, bleibt in passiver Seitenlage liegen, Lauf- und Atemkrämpfe. Rasseln in der Trachea. Blutiger Schaum wird aus der Nase entleert. Tod tritt nach 10 Minuten ein.

Sektionsbef und: Der Lungenbefund ist der gleiche wie bei den vorhergehenden Versuchstieren. Gewicht der Lunge 6 g.

Versuch 31. Meerschweinchen: Gewicht $200 \mathrm{~g}$. K.-G.

Injektionen von $0,2 \mathrm{~cm}$ Suprareninlösung, nach 2 Stunden von $0,3 \mathrm{ccm}$ pro $\mathrm{kg}$

Beobachtung: Macht sehr bald einen kranken Eindruck, liegt bewegungslos da, Atmung sehr beschleunigt, stoßweise. Nach $3 / 4$ Stunden wieder etwas erholt, aber noch krank. Am folgenden Morgen wieder normal. 
Versuch 32. Meerschweinchen. Gewicht $300 \mathrm{~g}$. K. -G.

Injektionen von $0,2 \mathrm{~cm}$ pro $\mathrm{kg} \mathrm{K}$.-G., nach 2 Stunden von $0,4 \mathrm{ccm}$ pro $\mathrm{kg}$

Beobachtung: Das Tier macht sehr bald einen kranken Eindruck, erholt sich aber in kurzer Zeit wieder.

Versuch 33. Meerschweinchen: Gewicht $540 \mathrm{~g}$.

Injektionen von $0,2 \mathrm{ccm}$, nach 3 Stunden von $0,3 \mathrm{ccm}$ pro $\mathrm{kg} \mathrm{K}$. -G.

Beobachtung: Das Tier zeigt leichte, aber sehr bald vorübergehende Erregungserscheinungen.

Versuch 34. Meerschweinchen: Gewicht $470 \mathrm{~g}$.

Versuchstier aus Versuch 1 nach $4^{1} \frac{1}{2}$ Stunden. Injektion von 0,34 ccm Suprareninlösung $1: 1000=0,73 \mathrm{ccm}$ pro $\mathrm{kg} \mathrm{K} .-\mathrm{G}$.

Fällt plötzlich ohne vorhergehende Erscheinungen auf die Seite, bleibt in passiver Seitenlage liegen. Krampfhafte Zuckungen treten auf. Blutiger Schaum wird aus der Nase entleert. Nach 6 Minuten tritt der Tod ein.

Sektionsbefund: Dieselben Iungenerscheinungen wie bei den vorhergehenden Versuchstieren. Gewicht der Lunge $11 \mathrm{~g}$.

Versuch 35. Meerschweinchen: Gewicht $630 \mathrm{~g}$.

Injektionen von $0,3 \mathrm{ccm}$, nach 2 Stunden von $0,2 \mathrm{ccm}$ pro $\mathrm{kg} \mathrm{K}$.-G.

Beobachtung: Kurze Zeit anhaltende Erregung, dann Rückkehr zur Norm.

Versuch 36. Meerschweinchen: Gewicht $610 \mathrm{~g}$.

Injektionen von $0,3 \mathrm{ccm}$, nach 3 Stunden von $0,2 \mathrm{ccm}$ pro $\mathrm{kg} \mathrm{K}$.-G.

Beobachtung: Wie in Versuch 35.

Versuch 37. Meerschweinchen: Gewicht $465 \mathrm{~g}$.

Versuchstier aus dem 2. Versuch. Nach $4^{1} / 2$ Stunden.

Injektion von $0,28 \mathrm{ccm}$ Suprareninlösung $1: 1000=0,6 \mathrm{ccm}$ pro $\mathrm{kg} \mathrm{K}$.-G.

Beobachtung: Nach 6 Minuten treten plötzlich heftige Krämpfe auf, dabei wird blutiger Schaum aus der Nase entleert. Unter zunehmenden Erstickungskrämpfen tritt nach 10 Minuten der Tod ein.

Sektionsbef und: Die Lungen finden sich in gleicher Weise verändert wie bei den vorhergehenden Versuchstieren. Gewicht der Lunge $9 \mathrm{~g}$.

Versuch 38. Meerschweinchen: Gewicht $390 \mathrm{~g}$.

Versuchstier aus dem 3. Versuch. Nach $4 \frac{1}{2}$ Stunden.

Injektion von $0,18 \mathrm{ccm}$ Suprareninlösung $1: 1000=0,45 \mathrm{com}$ pro $\mathrm{kg} \mathrm{K}$.G.

Beobachtung: Das Tier fällt ohne vorhergehende Anzeichen plötzlich auf die Seite, bleibt in passiver Seitenlage liegen, vereinzelte krampfartige aber spontane Bewegungen der Extremitäten. Nach 13 Minuten tritt der Tod ein.

Sektionsbef und: Die Lunge zeigt sich viel weniger ödematös wie die vorhergehenden, auch die Hämorrhagien sind weniger zahlreich. Herz nicht überlagert. Gewicht der Lunge $5 \mathrm{~g}$.

Versuch 39. Meerschweinchen: Gewicht $395 \mathrm{~g}$.

Versuchstier wie in Versuch 5. Nach 24 Stunden.

Injektion von $0,2 \mathrm{ccm}$ Suprareninlösung $1: 1000=0,5 \mathrm{ccm}$ pro $\mathrm{kg} \mathrm{K}$.-G.

Beobachtung: Das Tier bietet keine besonderen Erscheinungen.

Versuch 40. Meerschweinchen: Gewicht $490 \mathrm{~g}$.

Versuchstier wie in Versuch 4. Nach 24 Stunden.

Injektion von $0,27 \mathrm{ccm}$ Suprareninlösung $1: 1000=0,55 \mathrm{ccm}$ pro $\mathrm{kg} \mathrm{K}$.G.

Beobachtung: Wie in Versuch 39.

Versuch 41. Meerschweinchen: Gewicht $490 \mathrm{~g}$.

Versuchstier wie in Versuch 40. Nach 24 Stunden. 
Injektion von $0,27 \mathrm{ccm}$ Suprareninlösung $1: 1000=0,5 \mathrm{~cm}$ pro $\mathrm{kg} \mathrm{K} .-\mathrm{G}$.

Beobachtung: Leichte, bald vorübergehende Erregung.

Versuch 42. Meerschweinchen: Gewicht $395 \mathrm{~g}$.

Versuchstier wie in Versuch 39. Nach 24 Stunden.

Injektion von $0,24 \mathrm{ccm}$ Suprareninlösung $1: 1000=0,6 \mathrm{ccm}$ pro $\mathrm{kg} \mathrm{K}$.-G.

Berbachtung: Sehr bald macht sich eine heftige Erregung bemerkbar. Die Atmung ist stark beschleunigt und stoßweise. Das Tier bleibt krank. Es zeigt sich eine leichte Lähmung der hinteren Extremitäten. Unter zunehmender Lähmung erfolgt der Tod nach 48 Stunden.

Sektionsbef und: Jie Sektion ergibt die typischen Lungenerscheinungen in weit geringerem Maße, als es bei den vorhergehenden Tieren der Fall war.

Versuch 43. Meerschweinchen: Gewicht $490 \mathrm{~g}$.

Versuchstier wie in Versuch 41. Nach 24 Stunden.

Injektion von $0,31 \mathrm{ccm}$ Suprareninlösung $1: 1000=0,63 \mathrm{ccm}$ pro $\mathrm{kg} \mathrm{K}$.G.

Beobachtung: Nach 3 Minuten Eintreten der Erregung. Das Tier versucht fortzulaufen, ist aber unsicher in seinen Bewegungen. Die Extremitäten gleiten aus, es fällt auf die Seite, bleibt in passiver Seitenlage liegen. Vereinzelte krampfhafte Bewegungen, blutiger Schaum wird nicht entleert. Nach 11 Minuten tritt der Tod ein.

Sektionsbefund: Die Lunge zeigt sich stark ödematös mit zahlreichen Hämorrhagién. Herz ist nicht überlagert. Gewicht der Lunge $7 \mathrm{~g}$.

Subcutane Atropin-Adrenalininjektion.

Versuch 44. Meerschweinchen: Gewicht $720 \mathrm{~g}$.

6,40 Uhr. Injektion von 2,0 ccm Atropinlösung $1: 10$.

7,00 Uhr. Injektion der gleichen Dosis. kg K.-G.

7,10 Uhr. Injektion von 0,7 ccm Suprareninlösung $1: 1000=1,0 \mathrm{ccm}$ pro

Beobachtung: Bietet zunächst keine von der Norm abweichenden Erscheinungen. Frst 55 Minuten nach der letzten Injektion zeigt das Tier gesteigerte Erregung. Die vorderen und hinteren Extremitäten sind fast vollständig gelähmt. Hat sich am nächsten Morgen wieder ganz erholt.

Versuch 45. Meersohweinchen: Gewicht $620 \mathrm{~g}$.

12,20 Uhr. Injektion von 1,8 ccm Atropinlösung $1: 10$.

$12,40 \mathrm{Uhr}$. Injektion von $1,5 \mathrm{ccm}$ derselben Lösung.

12,50 Uhr. Injektion von $0,9 \mathrm{ccm}$ Suprareninlösung $1: 1000=1,5 \mathrm{ccm}$ pro kg K.-G.

Beobachtung: Das Tier bietet nach der Adrenalininjektion die gleichen Erscheinungen wie die Versuchstiere mit den höheren der untertödlichen Subcutandosen, vor allem sind die Lähmungserscheinungen der hinteren Extremitäten besonders gut ausgeprägt. Hat sich nach 1 Stunde wieder so weit erholt, daß es langsam umherkriecht. Nach 5 Stunden wieder normal.

\section{Literaturverzeichnis.}

1. Ba yer, in: Organotherapie, herausgeg. von J a u reg g - B a y e r. Leipzig 1914. G. Thieme.

2. Beckers und Meessen, Gaz. méd. belge. 15. I. 1903.

3. v. Bra u n, Berliner Klin. 1904, S. 11.

4. Cybulski, Anz. d. Akad. d. Wissensch. in Krakau. 4. III. 1895. Zit. nach Szymonowicz. 
5. Eckert, Therapeut. Monatshefte 1909, S. 114.

6. Erb, Archiv f. experim. Pathol. u. Pharmakol. 53, 175. 1905.

7. Exner, Archiv f. experim. Pathol. u. Pharmakol. 50, 313. 1903.

8. Falta und Ircovič, Wiener klin. Wochenschr. 1909.

9. Fröhlich und Pick, Archiv f. experim. Pathol. u. Pharmakol. ri, 23. 1913.

10. v. Fürth, Hofm. Beiträge 4, 421 .

11. Gluzins ki, Gazeta lekarska 15, Nr. 15. 13. IV. 1895. Zit. nach Szy mo nowicz.

13. Lesage, Arch. Int. de Pharmakodyn. 13. 1904.

14. Loewe und Simon, diese Zeitschr. 6, Heft 5/6.

15. - Therap. Monatshefte 1918, Heft 3.

16. Loewi, in v. Noor dens Handb. d. Path. d. Stoffwechsels 2. 1907.

17. Kretsch mer, Archiv f. experim. Pathol. u. Pharmakol. 57, 423. 1907.

18. Oliver und Schäfer, Journ. of Physiol. 17 u. 18. 1895.

19. Ritzmann, Archiv f. experim. Pathol. u. Pharmakol. 61, 231. 1909.

20. Stra ub, Münch. med. Wochenschr. 1909.

21. Szymonowicz, Archiv f. d. ges. Physiol. 1896.

22. Zeigan, Therap. Monatshefte 1904, S. 193. 